\title{
Self inductance of a wire loop as a curve integral
}

\author{
Richard Dengler \\ Rohde\&Schwarz GmbH \& Co KG, 1GS2 \\ Mühldorfstr. 15, 81671 Munich, P.O.B. 801469 \\ *corresponding author, E-mail: richard.denglererohde-schwarz.com
}

\begin{abstract}
It is shown that the self inductance of a wire loop can be written as a curve integral akin to the Neumann formula for the mutual inductance of two wire loops. The only difference is that contributions where the two integration variables get too close to each other must be excluded from the curve integral and evaluated in detail. The contributions of these excluded segments depend on the distribution of the current in the cross section of the wire. They add to a simple constant proportional to the wire length. The error of the new expression is of first order in the wire radius if there are sharp corners and of second order in the wire radius for smooth wire loops.
\end{abstract}

\section{Introduction}

Electrical inductance plays a crucial role in power plants, transformers and electronic devices. The coefficients of self and mutual inductance required to quantitatively describe inductance belong to the field of magnetostatics. Calculating inductance coefficients with analytic techniques is, however, impossible except in simple cases. The mathematical reason for the difficulty is that the Laplace equation allows analytic solutions only for some symmetric constellations. There thus are only a few closed-form expressions for these coefficients. In practice one often is forced to use approximations, finite element methods or other numerical techniques. The situation simplifies when the current flows in thin wires. This situation is analogous to an electrostatic system of point charges, where electric field and electrostatic energy directly follow from the given charge distribution, while in a generic system charge and current distributions also are unknown at the outset.

The purpose of this article is to derive a new expression for the self inductance of a wire loop, giving self inductance as a curve integral similar to the Neumann formula for mutual inductance. The starting point is the expression

$$
W=\frac{\mu_{0}}{8 \pi} \int \frac{\mathbf{j}(\mathbf{x}) \cdot \mathbf{j}\left(\mathbf{x}^{\prime}\right)}{\left|\mathbf{x}-\mathbf{x}^{\prime}\right|} d^{3} x d^{3} x^{\prime}
$$

for the magnetic field energy of a system with current density $\mathbf{j}(\mathbf{x})$, where $\mu_{0}$ is the magnetic constant.[1] This expression essentially was already given by Neumann in 1845.[2] It resembles the expression for gravitational or electrostatic potential energy, the only new ingredient is the scalar product between the current elements.
For a current density $\mathbf{j}(\mathbf{x})=\sum I_{m} \mathbf{j}_{m}(\mathbf{x})$ corresponding to $N$ separate current loops with currents $I_{m}$ and normalized current densities $\mathbf{j}_{m}$ it follows

$$
\begin{aligned}
W & =\frac{\mu_{0}}{8 \pi} \sum_{m, n=1}^{N} I_{m} I_{n} \int \frac{\mathbf{j}_{m}(\mathbf{x}) \cdot \mathbf{j}_{n}\left(\mathbf{x}^{\prime}\right)}{\left|\mathbf{x}-\mathbf{x}^{\prime}\right|} d^{3} x d^{3} x^{\prime}(2) \\
& \stackrel{!}{=} \frac{1}{2} \sum_{m, n=1}^{N} L_{m, n} I_{m} I_{n} .
\end{aligned}
$$

If the currents flow in thin wires, then the integrals become curve integrals, and one immediately reads off the Neumann expression for mutual inductance of two (filamentary) current loops[2]

$$
L_{1,2}=\frac{\mu_{0}}{4 \pi} \oint \frac{d \mathbf{x}_{1} \cdot d \mathbf{x}_{2}}{\left|\mathbf{x}_{1}-\mathbf{x}_{2}\right|} .
$$

It is plausible that there exists a similar expression for the self inductance of a wire loop, but we were not able to find any hint in the literature. Formally one might read off from equation (2) an expression similar to equation (3), where the two closed curves coincide. But this cannot be correct, because $\left|\mathbf{x}-\mathbf{x}^{\prime}\right|$ now vanishes and the integral isn't defined. Instead we will prove

$$
L=\frac{\mu_{0}}{4 \pi}\left(\oint \frac{d \mathbf{x} \cdot d \mathbf{x}^{\prime}}{\left|\mathbf{x}-\mathbf{x}^{\prime}\right|}\right)_{\left|s-s^{\prime}\right|>a / 2}+\frac{\mu_{0}}{4 \pi} l Y+\ldots
$$

where $a$ denotes the wire radius and $l$ the length of the wire. The variable $s$ measures the length along the wire axis. The constant $Y$ depends on the distribution of the current in the cross section of the wire: $Y=0$ if the current flows in the wire surface, $Y=1 / 2$ when the current is homogeneous across the wire. The ellipses represents terms like $O\left(\mu_{0} a\right)$ and $O\left(\mu_{0} a^{2} / l\right)$, which are negligible for $l \gg a$.

In hindsight it is completely natural to use a cutoff of order $a$ in the curve integral. In fact, the exact value of this cutoff is arbitrary, because the contribution proportional to $l Y$ also depends on this cutoff. The simplest way to determine $Y$ would be to compare the expression with the self inductance of a long rectangle.

\section{Simple derivation}

Consider equation (2) with $N=1$ for a thin wire with circular cross section, radius $a$ and length $l$. Let $s$ denote 


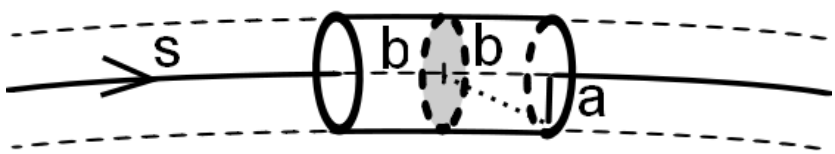

Figure 1: A section of a wire with radius $a$, with a segment of length $2 b$, and a plane perpendicular to the wire axis at the center of the segment.

the length along the axis of the wire. The planes perpendicular to the wire axis then define a projection from the bulk of the wire onto the axis, $\mathbf{x} \rightarrow s(\mathbf{x})$. Selecting a length scale $b$ satisfying $a \ll b \ll l$ allows to write $L=\left(\mu_{0} / 4 \pi\right)(\bar{L}+\widehat{L})$ with

$$
\begin{aligned}
\bar{L} & =\left(\int \frac{\mathbf{j}(\mathbf{x}) \mathbf{j}\left(\mathbf{x}^{\prime}\right)}{\left|\mathbf{x}-\mathbf{x}^{\prime}\right|} d^{3} x d^{3} x^{\prime}\right)_{\left|s-s^{\prime}\right|>b}, \\
\widehat{L} & =\left(\int \frac{\mathbf{j}(\mathbf{x}) \mathbf{j}\left(\mathbf{x}^{\prime}\right)}{\left|\mathbf{x}-\mathbf{x}^{\prime}\right|} d^{3} x d^{3} x^{\prime}\right)_{\left|s-s^{\prime}\right|<b} .
\end{aligned}
$$

The second part contains contributions from all point pairs $\left\{\mathbf{x}, \mathbf{x}^{\prime}\right\}$ with a distance along the axis smaller than $b$, the first the complement of this set ( $s$ is a cyclic quantity). For given $\mathbf{x}$ the planes at $s(\mathbf{x}) \pm b$ delimitate the points $\mathbf{x}^{\prime}$ contributing to the first or second integral, see figure (1). $\bar{L}$ now approximately becomes a curve integral and $\widehat{L}$ essentially consists of cylinders of length $2 b$.

The strategy then is to replace $\bar{L}$ with the curve integral and to explicitly evaluate the contribution of the cylinders in $\widehat{L}$. The cylinders are long in comparison to the radius because of $a \ll b$ and straight (at least most of them) because of $b \ll l$. Actually the only requirement for the lengths is $a \ll l$, the length $b=\sqrt{a l}$ then satisfies $a \ll b \ll l$. The approximation thus is exact in the limit $a \ll l$ except in special cases. Inserting $\widehat{L}_{0}$ for a straight segment from equation (A.4) in the appendix thus leads to

$L=\frac{\mu_{0}}{4 \pi}\left(\oint \frac{d \mathbf{x} \cdot d \mathbf{x}^{\prime}}{\left|\mathbf{x}-\mathbf{x}^{\prime}\right|}\right)_{\left|s-s^{\prime}\right|>b}+\frac{\mu_{0} l}{2 \pi}\left(\ln \left(\frac{2 b}{a}\right)+\frac{Y}{2}\right)+\ldots$

This expression cannot depend on the (more or less) arbitrary length scale $b$. The curve integral thus is ${ }^{1} \bar{L}(b)=$ const $-\frac{\mu_{0} l}{2 \pi} \ln \left(2 b / b_{0}\right)$. But $b$ is the only "short" length scale in the curve integral, and $\bar{L}(b)$ thus also is valid for $b=a / 2$. The expression (6) therefore doesn't change if one formally sets $b=a / 2$. Equation (6) now agrees with equation (4), but some questions remain.

First of all, how accurate is formula (4)? The curve integral is a purely geometric quantity with dimension "length" and order of magnitude $l$. Plausible expressions for the order of magnitude of the relative error are $a / l,(a / l)^{2}$ and $(a / R)^{2}$, with $R$ a typical curvature radius of the wire loop. Errors of this order normally are negligible (and also occur in the Neumann formula for mutual inductance). But the derivation of formula (4) is not as straightforward, and so what are the actual limits or exceptions?

\footnotetext{
${ }^{1}$ This also can easily be verified with an explicit calculation.
}

\section{Examples and comparison with exact self inductance}

To get an impression of the accuracy we have compared self-inductances calculated with the curve integral with the result of a numeric evaluation of the nominally 6 dimensional integral in equation (2). This integral becomes 4-dimensional if the currents flow in the wire surface (skin effect, $Y=0$ ), and the results below correspond to this skin effect case. The order of magnitudes of the error terms identified here are corroborated below in a more detailed derivation of formula (4).

\subsection{Straight segment}

The first example is a straight segment with length $c$ and complete skin effect. This of course isn't a closed circuit, but it might be an edge of a rectangle. The orthogonal edges of the rectangle don't interact with the segment because of the scalar product $\mathbf{j} \cdot \mathbf{j}^{\prime}$. What is missing for a rectangle are the interaction terms with the opposite edges (and the small contributions from the corners). In this case the volume integral (2) may even be evaluated analytically with the result

$$
L=\frac{\mu_{0}}{4 \pi}\left\{2 c\left[\ln \left(\frac{2 c}{a}\right)-1\right]+8 a / \pi-a^{2} / c+\ldots\right\},
$$

while the curve integral (4) leads to

$$
L_{c}(c)=\frac{\mu_{0}}{4 \pi}\left\{2 c\left[\ln \left(\frac{2 c}{a}\right)-1\right]+a\right\} .
$$

The difference is of order $O\left(\mu_{0} a\right)$, much smaller than $\mu_{0} c$ for $c \gg a$.

\subsection{Circular loop}

The next example is a ring with radius $R$. The curve integral (4) gives

$$
L_{c}=\mu_{0} R(\ln (8 R / a)-2+Y / 2)+\mu_{0} O\left(a^{2} / R\right) .
$$

This expression also may be found in the literature, derived with the help of elliptic functions and some approximations in a much more complicated way. The table displays the ratio of the exact inductance and $L_{c}$ for some values of $R / a$,

\begin{tabular}{l|lllll}
$R / a$ & 1 & 2 & 3 & 5 & 10 \\
\hline$L / L_{c}$ & 5.971 & 1.238 & 1.092 & 1.031 & 1.00756
\end{tabular}

The expression $L_{c}$ is more accurate than one might expect. It gives a reasonable approximation already for $R=3 a$, and the error roughly decays like $O\left(a^{2} / R^{2}\right)$.

\subsection{Rectangle}

This case is more complicated because in principle also the shape of the corners comes into play (curvature radius?). But the simplest thing to do is to evaluate the curve integral (4) for a rectangle with edges of length $c$ and $d$. Orthogonal edges decouple because of the scalar product $\mathbf{j} \cdot \mathbf{j}^{\prime}$, and the first contribution are the terms (7) for the four edges by 
themselves. The second contribution are the parts of the curve integral (4) with $\mathrm{x}$ on one edge and $\mathrm{x}^{\prime}$ on the opposite one. The condition $\left|s-s^{\prime}\right|>a / 2$ is irrelevant for these cross terms, and one easily obtains for parallel edges of length $c$ and distance $d$

$$
L_{c}(c, d)=\frac{\mu_{0}}{4 \pi}\left(4 \sqrt{c^{2}+d^{2}}-4 d-4 c \operatorname{asinh}(c / d)\right),
$$

and the sum together with the $Y$-term of equation (4) is

$$
\begin{aligned}
L_{c}= & \frac{\mu_{0}}{\pi}\left\{c \ln \frac{2 c}{a}+d \ln \frac{2 d}{a}-(c+d)(2-Y / 2)\right. \\
& \left.+2 \sqrt{c^{2}+d^{2}}-c \operatorname{asinh}(c / d)-d \operatorname{asinh}(d / c)+a\right\} .
\end{aligned}
$$

This expression also may be found in the literature, with sometimes a factor 2 at the $a$-term.[3] The table displays the ratio of the numerically evaluated self-inductance $L$ of a square with border length $c$ and corners with curvature radius $a$ and the curve integral $L_{c}$ for different border length $c$

\begin{tabular}{l|llll}
$c / a$ & 5 & 10 & 20 & 40 \\
\hline$L / L_{c}$ & 1.168 & 1.056 & 1.0205 & 1.00776 \\
$\left(L-L_{c}\right) / \mu_{0} a$ & 0.501 & 0.562 & 0.5871 & 0.5782 \\
$L / L_{c}^{\text {exact }}$ & 1.053 & 1.021 & 1.0080 & 1.0029
\end{tabular}

The curvature radius $a$ is minimal in that the centre of curvature lies on the inner border of the wire. It is remarkable that the absolute error nearly remains constant. The last row of the table displays the ratio of the exact self inductance and the exact curve integral (4) (with round corners), also evaluated numerically. This expression is a better approximation for small $c / a$, where the square with round corners degenerates to a ring.

\subsection{Equilateral triangle}

The curve integral (6) for an equilateral triangle with edge length $c$ consists of three times the expression (4) for the edges by themselves and three times the interaction energy $L_{c}(c, c, 120)$ of adjacent edges (with $s$ on one edge and $s^{\prime}$ on the other, see appendix Appendix C. There is no such interaction for rectangles because of the scalar product),

$$
L_{c}=\frac{\mu_{0}}{2 \pi} 3 c\left\{\ln \left(\frac{c}{a}\right)-1-\ln \frac{3}{2}\right\} .
$$

The table displays the ratio of the exact self-inductance $L$ of an equilateral triangle with border length $c$ and corners with curvature radius $a$ and the curve integral $L_{c}$ for different border length $c$,

\begin{tabular}{l|llll}
$c / a$ & 5 & 10 & 20 & 40 \\
\hline$L / L_{c}$ & 2.983 & 1.2534 & 1.0743 & 1.0264 \\
$\left(L-L_{c}\right) / \mu_{0} a$ & 0.966 & 1.0856 & 1.1288 & 1.1501 \\
$L / L_{c}^{\text {exact }}$ & 1.376 & 1.0931 & 1.0302 & 1.0110
\end{tabular}

The absolute error $\left(L-L_{c}\right) / \mu_{0}$ is nearly constant also here. The last row again is the ratio of the exact self inductance and the curve integral (4) with round corners, evaluated numerically.

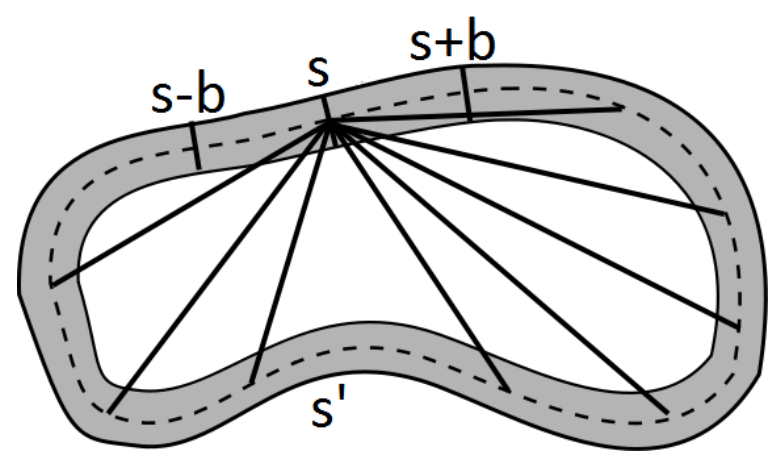

Figure 2: A wire loop and distances relevant for calculating the energy of a disc at $s$ relative to other points in the wire.

\subsection{Parallel wires}

For a loop consisting of infinitely long parallel wires the condition $a \ll l$ is perfectly met and the curve integral gives the exact self inductance even for minimal distance $d=2 a$,

$$
L_{c}=\frac{\mu_{0} l}{\pi}\left(\ln \frac{d}{a}+Y / 2\right) .
$$

This expression is the limiting case of the expression (8) for a long rectangle. The point is, that the contribution of the corners becomes negligible for a long rectangle, and that the replacement of the (circular symmetric) current by a line current doesn't change the magnetic field according to Ampere's law. Of course, the assumption of a circular symmetric current distribution gets wrong in the skin effect case if the wires are close to each other because of additional screening currents.

To summarize, formula (6) is rather accurate even for circuits with a linear extension as small as 20 times the wire radius, even if the circuit contains sharp corners.

\section{Error estimation}

According to equation (5) the self inductance may be written as $L=\left(\mu_{0} / 4 \pi\right)(\bar{L}+\widehat{L})$, where $\widehat{L}$ contains the short segments and $\bar{L}$ the complement. The self inductance $L$ of course doesn't depend on the arbitrary segment length $b$.

Let us now introduce some notation. We use a coordinate system $\{s, r, \phi\}$ in the wire where the length $s$ along the wire axis is cyclic with period $l$, and the coordinates $\{r, \phi\}$ describe planes perpendicular to the wire axis. The intersections of the planes and the wire are assumed to be circular, $0 \leqq r \leqq a$ and $0 \leqq \phi \leqq 2 \pi$. The volume element reads $d V=(1+r \cos \phi / R(s)) r d r d \phi d s$, where $R(s)$ denotes the curvature radius of the wire, and $\phi=0$ at the outer border of the wire (this is possible at least locally). The volume element also can be written as $d V=d s d \widetilde{A}$ with $d \widetilde{A}=(1+r \cos \phi / R(s)) d A$ and area element $d A=r d r d \phi$. The coordinates become cylindrical coordinates for straight wire segments, i.e. for $R=\infty$.

The current density $\mathbf{j}$ is normalized, that is $\int d A|\mathbf{j}|=\int d \widetilde{A}|\mathbf{j}|=1$. We will also need the radial 
moments

$$
a_{n}=\left\langle r^{n}\right\rangle=\int d \widetilde{A} r^{n}|\mathbf{j}|
$$

of the current distribution. In the skin effect case of course $a_{n}=a^{n}$.

One quantity of interest then is

$$
\bar{L}(s)=\int^{>} d s^{\prime} \int d \widetilde{A} d \widetilde{A}^{\prime} \frac{\mathbf{j}(s, r, \phi) \cdot \mathbf{j}\left(\mathbf{x}^{\prime}\right)}{\left|\mathbf{x}(s, r, \phi)-\mathbf{x}\left(s^{\prime}, r^{\prime}, \phi^{\prime}\right)\right|},
$$

the energy of the current in the plane at $s=0$ with respect to the current at $\left|s^{\prime}-s\right|>b$, see figure (2). The symbol ' $>$ ' is an abbreviation for a step function factor $\theta\left(\left|s^{\prime}-s\right|-b\right)$. To obtain $\bar{L}$ from $\bar{L}(s)$ requires to integrate over $s$. The curve integral

$$
\bar{L}_{\gamma}(s)=\oint^{>} d s^{\prime} \frac{\cos \left(\mathbf{j}(s), \mathbf{j}\left(s^{\prime}\right)\right)}{\left|\mathbf{x}(s, 0,0)-\mathbf{x}\left(s^{\prime}, 0,0\right)\right|},
$$

is an approximation for $\bar{L}(s)$. Similarly we write the short segment around the plane at $s=0$ as

$$
\widehat{L}(s)=\int^{<} d s^{\prime} \int d \widetilde{A} d \widetilde{A}^{\prime} \frac{\mathbf{j}(s, r, \phi) \cdot \mathbf{j}\left(s^{\prime}, r^{\prime}, \phi^{\prime}\right)}{\left|\mathbf{x}(s, r, \phi)-\mathbf{x}\left(s^{\prime}, r^{\prime}, \phi^{\prime}\right)\right|},
$$

These definitions allow to write

$$
\begin{aligned}
\frac{4 \pi}{\mu_{0}} L(s) & =\bar{L}(s)+\widehat{L}(s)=\left(\bar{L}_{\gamma}+\widehat{L}_{\gamma}\right)(s) \\
& +\left(\bar{L}-\bar{L}_{\gamma}+\hat{P}_{0}\right)(s)+\left(\widehat{L}-\widehat{L}_{0}\right)(s)
\end{aligned}
$$

where we have added and subtracted the curve integral $\bar{L}_{\gamma}(s)$, the segment integral $\widehat{L}_{0}(s)$ for a straight segment from equation (A.4) and the approximation $\widehat{L}_{\gamma}(s)=$ $2(\ln (2 b / a)+Y / 2)$ for $\widehat{L}_{0}(s)$. The first bracket in equation (12) now is formula (6), and the two other brackets thus represent the error. The second bracket contains the difference of the volume and the curve integral (for $\left|s^{\prime}-s\right|>b$ ) plus the power series $\hat{P}_{0}=\widehat{L}_{0}-\widehat{L}_{\gamma}$ from equation (A.4), the third bracket is the difference of the actual segment integral (11) and the segment integral for a straight segment. It is evident that the error becomes small in suitable limits, and we now want to determine the order of magnitude of the error.

\subsection{Smooth current loops}

We first consider smooth current loops, that is current loops with a minimal curvature radius $R$ comparable with the system size. We also assume that the loop returns immediately and doesn't touch itself anywhere in between. Such complications are considered below.

The coordinates $\mathbf{x}$ and $\mathbf{x}^{\prime}$ in the integral (9) above may be expanded like $\mathbf{x}=\mathbf{x}(s, 0,0)+\mathbf{x}_{1}(s, r, \phi)$, and thus

$$
\begin{aligned}
\mathbf{x}-\mathbf{x}^{\prime} & =\mathbf{x}_{s, s^{\prime}}+\mathbf{x}_{1}-\mathbf{x}_{1}^{\prime} \\
\left(\mathbf{x}-\mathbf{x}^{\prime}\right)^{2} & =x_{s, s^{\prime}}^{2}+2 \mu x_{s, s^{\prime}}+\nu^{2}
\end{aligned}
$$

with $\mathbf{x}_{s, s^{\prime}}=\mathbf{x}(s, 0,0)-\mathbf{x}\left(s^{\prime}, 0,0\right)$ the distance of the projections onto the axis and $\left|\mathbf{x}_{1}\right|$ and $\left|\mathbf{x}_{1}^{\prime}\right|$ of order $O(a)$. The abbreviations are

$$
\begin{aligned}
\mu & =\widehat{\mathbf{x}}_{s s^{\prime}} \cdot \mathbf{x}_{1}+\widehat{\mathbf{x}}_{s^{\prime} s} \cdot \mathbf{x}_{1}^{\prime}, \\
\nu^{2} & =\left(\mathbf{x}_{1}-\mathbf{x}_{1}^{\prime}\right)^{2} .
\end{aligned}
$$

The procedure now is to use the multipole expansion

$$
\begin{aligned}
\frac{1}{\left|\mathbf{x}-\mathbf{x}^{\prime}\right|}= & \frac{1}{x_{s, s^{\prime}}}-\frac{\mu}{x_{s, s^{\prime}}^{2}}+\frac{1}{2 x_{s, s^{\prime}}^{3}}\left(3 \mu^{2}-\nu^{2}\right) \\
& -\frac{1}{2 x_{s, s^{\prime}}^{4}}\left(5 \mu^{3}-3 \mu \nu^{2}\right)+ \\
& \frac{1}{8 x_{s, s^{\prime}}^{5}}\left(35 \mu^{4}-30 \mu^{2} \nu^{2}+3 \nu^{4}\right)+O\left(\frac{a^{5}}{x_{s, s^{\prime}}^{6}}\right) .
\end{aligned}
$$

This expansion converges for $x_{s, s^{\prime}} \geqq\left|\mathbf{x}_{1}^{\prime}-\mathbf{x}_{1}\right|$, the coefficients are the coefficients of the Legendre polynomials. Inserting the leading monopole term $1 / x_{s, s^{\prime}}$ of equation (13) into equation (9) reproduces the curve integral $\bar{L}_{\gamma}(s)$. The higher multipole terms describe the difference between the volume integral $\bar{L}(s)$ and the curve integral. The $s^{\prime}$-integral in the multipole terms converges and the difference thus is mainly a local quantity. The nominal order of magnitude of the multipole terms $\bar{L}_{n}$ is $a^{n} / b^{n}, n \geqq 1$. With a length $b=\sqrt{a R}$ the order of magnitude becomes $(a / R)^{n / 2}$, and the expansion up to the hexadecupole $(n=4)$ is needed to get the $(a / R)^{2}$ approximation.

The volume element is

$$
\left(1+\frac{r \cos \phi}{R(s)}\right) r d r d \phi d s=\left(1+\frac{\mathbf{x}_{1} \cdot \mathbf{R}(s)}{R^{2}(s)}\right) r d r d \phi d s,
$$

where $\mathbf{R}(s)$ is the local curvature radius vector. Integrals over $\phi$ and $\phi^{\prime}$ then may be evaluated with the help of

$$
\begin{aligned}
\left\langle\mathbf{x}_{1}\right\rangle & =0 \\
\left\langle\left(\mathbf{x}_{1}\right)_{m}\left(\mathbf{x}_{1}\right)_{n}\right\rangle & =r^{2} P_{m, n} / 2
\end{aligned}
$$

where $P_{m, n}$ is the projection operator projecting onto the plane perpendicular to the wire axis. This implies $\mathbf{P} \cdot \mathbf{R}=$ $\mathbf{R}$. Inserting now the multipole expansion (13) into the volume integral (9) generates an expansion of the difference of the volume and the curve integral in the region $\left|s^{\prime}\right|>b$.

Dipole: The voltage drops by the same amount along inner and outer border along curved parts of the loop. Electric field and current density thus are larger at the inner border, and the current density thus depends on $r$ and $\varphi$. This $\varphi$-dependence of the current density compensates the $\varphi$-dependence $1+r \cos (\varphi) / R$ from the volume element. The simple result then is: there is no dipole contribution. The factors $\cos \varphi$ and $\cos \varphi^{\prime}$ from $\mu$ give 0 after integration over the angles. There are in fact short transition regions between straight and curved parts of the loop where the current distribution changes from uniform to non-uniform, but this only leads to corrections of higher order.

Quadrupole: After integration over the angles $\phi$ and the radial coordinates $r$ there remains 


$$
\begin{aligned}
\bar{L}_{2}(s)= & a_{2} \oint^{>} d s^{\prime}\left[\frac{3}{4}\left(1-\left(\widehat{\mathbf{n}} \cdot \widehat{\mathbf{x}}_{s, s^{\prime}}\right)^{2}\right)\right. \\
& \left.+\frac{3}{4}\left(1-\left(\widehat{\mathbf{n}}^{\prime} \cdot \widehat{\mathbf{x}}_{s, s^{\prime}}\right)^{2}\right)-1\right] \frac{\cos \alpha}{x_{s, s^{\prime}}^{3}},
\end{aligned}
$$

where $\widehat{\mathbf{n}}$ denotes a unit vector in the direction of the wire axis and the expression $\mathbf{P}=1-\widehat{\mathbf{n}} \widehat{\mathbf{n}}$ for the projection operator was used. In $\bar{L}_{2}(s)$ one may recognize $1-\left(\widehat{\mathbf{n}} \cdot \widehat{\mathbf{x}}_{s, s^{\prime}}\right)^{2}=\sin ^{2} \psi$ and $1-\left(\widehat{\mathbf{n}}^{\prime} \cdot \widehat{\mathbf{x}}_{s, s^{\prime}}\right)^{2}=$ $\sin ^{2} \psi^{\prime}$, where $\psi$ denotes the angle between the distance vector $\mathbf{x}_{s, s^{\prime}}$ and the wire axis. With $\psi^{\prime}=\psi=2 \alpha=$ $(s-s)^{\prime} / 2 R$ for a smooth current loop there remains an error $\left(a^{2} / R^{2}\right) \ln (R / b)$. The $-a_{2} / b^{2}$ term (from the -1 ) is peculiar. With the choice $b=\sqrt{a R}$ it would be of order $a / R$, but it gets cancelled against the leading term of the power series $\hat{P}_{0}$ of equation (12). This of course is the reason for combining $\hat{P}_{0}$ with the multipole expansion in equation (12): for a straight segment the formula is exact, and the third bracket in equation (12) vanishes. The multipole expansion together with $\hat{P}_{0}$ thus also vanishes for $R=\infty$.

Oktupole: The oktupole contributes at most a term of order $a^{3} / b^{3}$. But the $\cos \phi$ and $\sin \psi$ from the odd power of $\mu$ make the actual contribution smaller than the expected $\sim a^{2} / R^{2}$.

Hexadecupole: The hexadecupole term is of order $a^{4} / b^{4} \sim a^{2} / R^{2}$ and its leading part must be kept. The $\mu$ factors contain a factor $\sin \psi \sim b / R$ and may be dropped. The $\nu^{4}$ leads to

$$
\bar{L}_{4}(s)=\frac{3}{8} \oint^{>} d s^{\prime}\left[2 a_{4}+a_{2}^{2}\left(3+\cos ^{2} \alpha\right)\right] \frac{\cos \alpha}{x_{s, s^{\prime}}^{5}} .
$$

The factors $\cos \alpha$ may be replaced with 1 because $\alpha=$ $\left(s^{\prime}-s\right) / R$ is small. The integral converges and contributes an error like $a^{2} / R^{2}$. But this term gets cancelled by the second term of the power series $\hat{P}_{0}$ from equation (A.4).

For smooth current loops it finally follows

$$
\frac{4 \pi}{\mu_{0}} L(s)=\left(\bar{L}_{\gamma}+\widehat{L}_{\gamma}\right)+O\left(\frac{a^{2}}{R} \ln \frac{R}{b}\right)+\left(\widehat{L}-\widehat{L}_{0}\right),
$$

where the first bracket on the r.h.s is formula (6), evaluated with $b=\sqrt{a R}$. The remaining segment integral $\widehat{L}$ for a segment with curvature radius $R$ is evaluated in appendix Appendix B. The result (B.1) contributes another logarithmic error $\left(a^{2} / R^{2}\right) \ln (R / b)$, and a larger error of order $b^{2} / R^{2} \sim a / R$. We now drop all errors of order $O\left(\left(a^{2} / R\right) \ln (R / b)\right)$ and $O\left(a^{2} / R\right)$ from the multipole expansion and the curved segment.

As in the simple derivation of the formula above one may notice that there is no small lenght scale in the formula $\bar{L}_{\gamma}+\widehat{L}_{\gamma}$ and its $b$-dependence is under control for all $b<\sqrt{a R}$ and given by (B.2). It contains a "large" $b^{2} / R^{2} \sim a / R$ term. The essential point now is that this $b$-dependence of the formula exactly absorbs the remaining large error from $\widehat{L}$ (the cancellation comes about, because $b$ is the lower limit in the curve integral and the upper limit in the volume integral). Nothing therefore changes if we now set $b=a / 2$, except that there only remains formula (6) evaluated for $b=a / 2$ and small terms of order $O\left(\left(a^{2} / R\right) \ln (R / a)\right)$ and $O\left(a^{2} / R\right)$.

For a circular loop the leading error terms can even be evaluated in closed form. The results for the selfinductances in the skin effect and volume current case are

$$
\begin{aligned}
& L_{S}=\mu_{0} R\left\{\left(1+\frac{3}{4} \frac{a^{2}}{R^{2}}\right) \ln \left(\frac{8 R}{a}\right)-2-\frac{3}{2} \frac{a^{2}}{R^{2}}\right\}, \\
& L_{V}=\mu_{0} R\left\{\left(1+\frac{3}{8} \frac{a^{2}}{R^{2}}\right) \ln \left(\frac{8 R}{a}\right)-\frac{7}{4}-\frac{2}{3} \frac{a^{2}}{R^{2}}\right\} .
\end{aligned}
$$

The corrections perfectly agree with a numeric evaluation of the 6- or 4-dimensional integrals, but strongly disagree with expressions in the literature.[4] The reason appears to be that these calculations assume a constant current distribution in the surface or cross section of the wire, wrong just in the curved parts of the loop.

\subsection{Current loops with sharp corners}

The errors originate from the curved parts of the current loop, and for current loops with sharp corners one may expect larger errors. A simple way to construct such loops is to insert straight segments into a circular loop. It was shown above that the absolute error for a circular loop with radius $R$ is of order $O\left(\mu_{0} a^{2} / R\right)$. This estimation is valid even for minimal curvature radius $R=a$ (the inductance has dimension length $\times \mu_{0}$ and $a$ is the only available length for such loops). Since the loop with inserted straight segments is better approximated by the curve integral the absolute error can only be smaller. The formal reason is that a filamentary straight segment generates the same magnetic field as the actual axially symmetric current distribution.

The inductance of a loop of extension $l \gg a$ generally is of order $O\left(\mu_{0} l \cdot \ln (l / a)\right)$. The ratio with the absolute error leads to a generic estimation of the relative error of formula (4)

$$
\Delta L / L=\sum_{n} O\left(\frac{a^{2}}{l R_{n}}\right),
$$

where the index $n$ enumerates the corners and the logarithmic factor is neglected. We have allowed here corners with different curvature radius $R_{n}$. The special case with curvature radii of order $O(l)$ leads back to the estimation of the error for smooth current loops above. Sharp corners with curvature radius $R=O(a)$ contribute a relative error of order $O(a / l)$. Corners with a small angle of course should get a smaller weight in the sum. This estimation could be made more rigorous, but the procedure only is circumstantial and of no interest here.

\subsection{Current loops not closing immediately}

The error estimations above fail if the current loop comes close to itself before it actually closes, that is for tight spirals, coils or something like that (the estimation $x_{s, s^{\prime}} \sim$ $\left|s^{\prime}-s\right|$ gets invalid). In this case the replacement of the actual current with a filamentary current generates additional 
errors at these positions. But this isn't specific for equation (4), exactly the same (small) errors are contained in the Neumann formula for mutual inductance.

The error can easily be estimated, because the condition $\left|s^{\prime}-s\right|>b$ is irrelevant if $s^{\prime}$ is on one winding and $s$ on another. A simple possibility is to consider as a worst case scenario two current loops of radius $R$ a distance $d>2 a$ on top of each other. The error comes from the multipole expansion (13) valid everywhere now, and it is a simple matter to estimate the integrals. The order of magnitude of the relative error is $O\left(a^{2} / R d\right)$, small except for small curvature radius and small distance (both of order $O(a)$ ).

\section{Conclusion}

The curve integral (4) for the self inductance of a wire loop is only a little bit more complicated than the Neumann formula for the mutual inductance of two wire loops. The exact expression for self inductance is a 6-dimensional integral with a logarithmic divergence and several length scales. Nevertheless clear statements follow for the accuracy of formula (4), for loops consisting of straight segments as well as for smooth loops. The error originates from the curved parts of the loop, and is of order $\mu_{0} a$ or $\mu_{0} a^{2} / l$, negligible for most practical purposes. The leading error presumably even might be given as a sum over the corners for loops consisting of straight segments or as additional curve integrals for smooth current loops (see the quadrupole contribution above). The techniques used for error estimation also may be used for the Neumann formula.

The self inductance curve integral can be evaluated analytically in many cases, for instance for current loops consisting of coplanar straight segments (not a new result; see also appendix Appendix C and Appendix D). But equation (4) is valid for arbitrary curves, and the numerical evaluation of two-dimensional integrals with a computer program is a breeze with appropriate numerical libraries. The information for the self inductance is contained in the curve spanned by the current loop, and any self inductance calculation at least requires a double integral along the curve. Simpler methods or estimations based on "partial inductance" or only the magnetic flux miss this point and only may work in special cases. The fact that two coinciding points cause problems in self inductance calculations is well known and has been circumvented in several ways, for instance by distributing the current onto two filamentary loops. But no systematic approximations can be obtained in this way.

Current distributions which are not circular symmetric also lead to formula (4), with a cutoff and a constant $Y$ depending on the current distribution. An example are circuits consisting of coplaner flat strips of width $w$. The self inductance of such circuits is given in the accuracy described above by formula (4) with $a=w$ and $Y=3$.

\section{Appendix A. Contribution from straight segments of length $2 b$}

The contribution $\widehat{L}$ to the self inductance in equation (5) is due to the interaction of the current in the plane $s$ with the current in all planes $s^{\prime}$ with $\left|s^{\prime}-s\right|<b$. This value depends on the current distribution in the wire and on the wire geometry within the segment $[s-b, s+b]$, but may be evaluated if the segment is straight or slightly curved. This $s$-dependent value still is to be integrated over all $s$.

To get an approximation for $\widehat{L}$ in the straight wire case use cylinder coordinates with a length $s$ along the axis and area element $d A=r d r d \phi$ (see figure (1)). This leads to

$$
\begin{aligned}
\widehat{L}_{0} & =\oint d s \widehat{L}(s) \\
\widehat{L}_{0}(s) & =\left(\int \frac{\mathbf{j}(r) \mathbf{j}\left(r^{\prime}\right)}{\left|\mathbf{x}(s, r, \phi)-\mathbf{x}^{\prime}\right|} d s^{\prime} d A^{\prime} d A\right)_{\left|s\left(\mathbf{x}^{\prime}\right)-s\right|<b} .
\end{aligned}
$$

In the latter integral $\mathrm{x}$ extends over the plane through the centre of a cylindrical segment, $\mathbf{x}^{\prime}$ extends over the complete segment. The integral $\widehat{L}_{0}(s)$ of course is independent of $s$. The integral over $s^{\prime}$ (from $-b$ to $b$ ) may be performed using $\left|\mathbf{x}-\mathbf{x}^{\prime}\right|^{2}=N^{2}+s^{\prime 2}, N^{2}=r^{2}+r^{\prime 2}-$ $2 r r^{\prime} \cos \left(\phi-\phi^{\prime}\right)$,

$$
\begin{array}{r}
\widehat{L}_{0}(0)=2 \int d A d A^{\prime} \mathbf{j}(r) \mathbf{j}\left(r^{\prime}\right) \operatorname{asinh}(b / N) \\
=2 \int d A d A^{\prime} \mathbf{j}(r) \mathbf{j}\left(r^{\prime}\right)\left\{\ln \frac{2 b}{a}-\ln \frac{N}{a}+A_{1}\left(\frac{N}{b}\right)+\ldots\right\}
\end{array}
$$

In the second line the expansion

$$
\begin{aligned}
\operatorname{asinh}(x) & =\ln (2 x)+A_{1}(1 / x) \\
A_{1}(x) & =\sum_{n=1}^{\infty} \frac{1 \cdot 3 \ldots(2 n-1)}{2 \cdot 4 \ldots 2 n} \frac{(-1)^{n+1}}{2 n} x^{2 n} \\
& =x^{2} / 4-3 x^{4} / 32+\ldots
\end{aligned}
$$

was used. The expansion converges because of $N=$ $O(a) \ll b$. It doesn't matter which $\phi^{\prime}$ occurs in the $\phi$-integral and thus we now set $\phi^{\prime}=0$. Because of $\int d A|\mathbf{j}|=1$ the leading term simply becomes $2 \ln (2 b / a)$. The second term follows from $\ln (N / a)=$ $\frac{1}{2} \ln \left(\rho^{2}+\rho^{\prime 2}-2 \rho \rho^{\prime} \cos \phi\right)$ with $\rho=r / a$ and $\rho^{\prime}=r^{\prime} / a$ and

$$
\frac{1}{2 \pi} \int_{0}^{2 \pi} \ln \left(\rho^{2}+\rho^{\prime 2}-2 \rho \rho^{\prime} \cos \phi\right) d \phi=2 \ln \left(\rho_{>}\right)
$$

where $\ln \left(\rho_{>}\right)=\theta\left(\rho-\rho^{\prime}\right) \ln \rho+\theta\left(\rho^{\prime}-\rho\right) \ln \rho^{\prime}$. This term thus vanishes in the skin effect case where the current differs from 0 only for $\rho=\rho^{\prime}=1$. The current density in the constant current case is $j(r)=1 /\left(\pi a^{2}\right)$ and the second term becomes

$$
-\frac{(2 \pi)^{2}}{\pi^{2}} 2 \int_{0}^{1} d \rho \rho \int_{0}^{1} d \rho^{\prime} \rho^{\prime} \ln \left(\rho_{>}\right)=1 / 2 .
$$


A rapidly convergent expansion for $b \gg a$ thus is

$$
\begin{array}{lcl}
\widehat{L}_{0}(0) & = & \widehat{L}_{\gamma}(0)+\widehat{P}_{0}(0), \\
\widehat{L}_{\gamma}(0) & = & 2 \ln (2 b / a)+Y, \\
\widehat{P}_{0}(0)=\left\langle 2 A_{1}\left(\frac{N}{b}\right)\right\rangle= & \frac{a_{2}}{b^{2}}-\frac{3}{8 b^{4}}\left(a_{4}+2 a_{2}^{2}\right)+O\left(\frac{a^{6}}{b^{6}}\right)
\end{array}
$$

with $Y=1 / 2$ for a constant current distribution and $Y=0$ in the skin effect case.

\section{Appendix B. Contribution from a curved segment}

The goal is to evaluate the integral $\widehat{L}(0)$ from equation (11) for a segment of length $2 b$ and constant curvature radius $R$,

$\widehat{L}_{R}(0)=\int \frac{d s^{\prime} d \widetilde{A} d \widetilde{A}^{\prime}}{\left|\mathbf{x}(0, r, \phi)-\mathbf{x}^{\prime}\right|} \theta\left(b-\left|s^{\prime}\right|\right) j(r) j\left(r^{\prime}\right) \cos \left(\frac{s^{\prime}}{R}\right)$.

The distance up to order $O\left(R^{-2}\right)$ follows from

$$
\begin{aligned}
\left(\mathbf{x}-\mathbf{x}^{\prime}\right)^{2} & \cong s^{\prime 2}+N^{2}+q, \\
N^{2} & =r^{2}+r^{\prime 2}-2 r r^{\prime} \cos \left(\phi-\phi^{\prime}\right), \\
q & =s^{\prime 2}\left[\frac{r^{\prime} \cos \phi^{\prime}+r \cos \phi}{R}+\frac{r r^{\prime}}{R^{2}} \cos \phi \cos \phi^{\prime}\right] \\
& -s^{\prime 4} /\left(12 R^{2}\right)+s^{\prime 6} /\left(360 R^{4}\right)+\ldots
\end{aligned}
$$

Expanding in $q$ gives

$$
\frac{1}{\left|\mathbf{x}-\mathbf{x}^{\prime}\right|}=\frac{1}{\left(s^{\prime 2}+N^{2}\right)^{1 / 2}}-\frac{q / 2}{\left(s^{\prime 2}+N^{2}\right)^{3 / 2}}+\frac{3 q^{2} / 8}{\left(s^{\prime 2}+N^{2}\right)^{5 / 2}}+\ldots
$$

The $N$ in the denominators is negligible for $b \gg a$ and the elementary integrals lead to

$$
\widehat{L}_{R}(0)-\widehat{L}_{0}(0)=-\frac{11}{24} \frac{b^{2}}{R^{2}}+O\left(\frac{a_{2}}{R^{2}} \ln \frac{b}{a}\right) .
$$

The term of order $O\left(a_{2} / R^{2}\right)$ contributes to the error of the self inductance formula with the expected order of magnitude. It is essential however, that the $O\left(b^{2} / R^{2}\right)$ term, which is of order $a / R$ because of $b=\sqrt{a R}$, cancels against a contribution from the curve integral $\bar{L}_{\gamma}(0)$ from equation (10).

To verify this cancellation start with

$$
\frac{d}{d b} \bar{L}_{\gamma}(0)=\frac{-\cos (\mathbf{j}(0), \mathbf{j}(b))}{|\mathbf{x}(0)-\mathbf{x}(b)|}-\frac{-\cos (\mathbf{j}(0), \mathbf{j}(-b))}{|\mathbf{x}(0)-\mathbf{x}(-b)|} .
$$

Inserting $\left|x-x^{\prime}\right|=2 R \sin b / 2 R$ and $\cos (\mathbf{j}(0), \mathbf{j}(b))=$ $\cos b / r$ for a section with curvature radius $R$ gives

$$
\frac{d}{d b} \bar{L}_{\gamma}(0)=\frac{-2}{b}\left(1-\frac{11}{24}\left(\frac{b}{R}\right)^{2}+\ldots\right),
$$

with integral

$$
\bar{L}_{\gamma}(0)=\text { const }-2 \ln \frac{2 b}{a}+\frac{11}{24} \frac{b^{2}}{R^{2}}+\ldots
$$

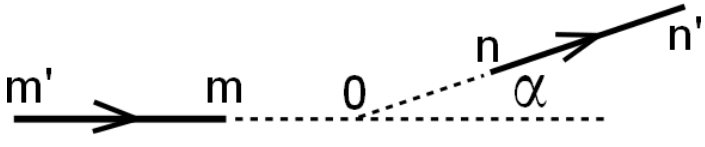

Figure 3: Two non-adjacent coplanar straight segments. The coordinates $m, m^{\prime}$ and $n, n^{\prime}$ measure the distance of the end points from the intersection of the segment extensions.

\section{Appendix C. Curve integral for adjacent straight segments}

For completeness we display here the curve integral contribution to the self inductance from adjacent straight segments of length $c$ and $d$ with an angle $\alpha$ between the currents,

$$
\begin{aligned}
L_{\gamma}(c, d, \alpha)= & \frac{\mu_{0}}{2 \pi} \cos (\alpha)\left\{c \operatorname{asinh} \frac{d+c \cos \alpha}{c \sin \alpha}\right. \\
& +d \operatorname{asinh} \frac{c+d \cos \alpha}{d \sin \alpha}-(c+d) \operatorname{asinh} \frac{\cos \alpha}{\sin \alpha} \\
& \left.-\frac{2 b}{\sqrt{1(1-\cos \alpha)}} \operatorname{asinh} \frac{1-\cos \alpha}{\sin \alpha}\right\} .
\end{aligned}
$$

For each corner such a term is to be added to the contribution (7) of the (straight) segments by themselves. The $b$-term is of order $O(a)$ for $b=a / 2$ and normally may be neglected.

\section{Appendix D. Curve integral for non-adjacent coplanar straight segments}

What then is missing for calculating the self inductance of a loop consisting of arbitrary coplanar straight segments is the mutual contribution from non-adjacent straight segments, see figure (3).

$$
\begin{aligned}
L\left(m, m^{\prime}, n, n^{\prime}, \alpha\right) & =\frac{\mu_{0}}{2 \pi}\left\{A\left(m^{\prime}, n^{\prime}, n, \alpha\right)\right. \\
& +A\left(n^{\prime}, m^{\prime}, m, \alpha\right)+A\left(m, n, n^{\prime}, \alpha\right) \\
& \left.+A\left(n, m, m^{\prime}, \alpha\right)\right\}, \\
A(w, u, v, \alpha) & =w \operatorname{asinh}\left(\frac{u+w \cos \alpha}{w \sin \alpha}\right) \cos \alpha . \\
& -w \operatorname{asinh}\left(\frac{v+w \cos \alpha}{w \sin \alpha}\right) \cos \alpha .
\end{aligned}
$$

This leads to an unwieldy expression already for a hexagon, but the calculation of the self inductance of such loops is a matter of algebra and geometry (not a new result).

\section{References}

[1] J.D. Jackson, "Classical Electrodynamics", p. 261. Wiley 1975.

[2] F. E. Neumann, "Allgemeine Gesetze der inducirten elektrischen Ströme”. Abhandlungen der Königlichen 
Akademie der Wissenschaften zu Berlin, aus dem Jahre 1845: 1-87, 1847.

[3] E.B. Rosa, The self and mutual inductances of linear conductors. Bulletin of the Bureau of Standards 4 (2) (1907) 301-344.

[4] E.B. Rosa and F.W. Grover, Formulas and tables for the calculation of mutual and self-inductance. Scientific papers of the bureau of standards (1916). Government printing office, Washington 1948. 